Supporting Information for

\title{
Radical SAM Enzyme QmpB Installs Two 9-Membered Ring Sactionine Macrocycles During Biogenesis of a Ribosomal Peptide Natural Product
}

Alessio Caruso ${ }^{\dagger}$ and Mohammad R. Seyedsayamdost ${ }^{\dagger, \ddagger, *}$

† Department of Chemistry, Princeton University, Princeton, NJ 08544

‡Department of Molecular Biology, Princeton University, Princeton, NJ 08544

*Corresponding author. E-mail address: mrseyed@princeton.edu

\section{Table of Contents}

Strains and plasmids (Table S1)

HR-MS \& HR-MS/MS results for QmpB substrates/products (Tables S2-S5)

UV-vis absorption spectrum of reconstituted QmpB (Figure S1)

Sequence comparison of QmpB with SuiB and CteB (Figure S2)

S7

QmpB-catalyzed formation of 5'-dA in the absence of QmpA (Figure S3)

HR-MS/MS analysis of C28S-NxxcA after reaction with NxxcB (Figure S4)

HR-MS analysis of ${ }^{13} \mathrm{C}-\mathrm{QmpA}$ after reaction with QmpB (Figure S5) 
Table S1. Strains and plasmids used and generated in this study.

\begin{tabular}{|c|c|c|}
\hline Strain & Plasmid Purpose & Source \\
\hline E. coli DH5a & Host strain for cloning & NEB \\
\hline E. coli BL21(DE3) & Host strain for expression & NEB \\
\hline pET-28b(+) & $\mathrm{Kan}^{\mathrm{R}}$, Expression vector & Novagen \\
\hline pDB1282 & $\mathrm{Amp}^{\mathrm{R}}$, isc operon & $\begin{array}{l}\text { Profs. D. Dean } \\
\text { \& S. Booker }\end{array}$ \\
\hline pET-28b(+)_qmpB & $\begin{array}{c}\mathrm{Kan}^{\mathrm{R}} \text {, Expression of } \\
\text { QmpB }\end{array}$ & This study \\
\hline $\begin{array}{l}\text { E. coli BL21(DE3) + pET- } \\
28 \mathrm{~b}(+) \_q m p B+p D B 1282\end{array}$ & Expression of QmpB & This study \\
\hline
\end{tabular}


Table S2. HR-MS data for QmpA substrate peptides and QmpB reaction products.

\begin{tabular}{|c|c|c|c|}
\hline \multirow{2}{*}{ Peptide } & \multicolumn{3}{|c|}{ Substrate } \\
\hline & {$[\mathrm{M}+3 \mathrm{H}]^{3+}{ }_{\text {calc }}$} & {$[\mathrm{M}+3 \mathrm{H}]^{3+}{ }_{\text {obs }}$} & $\Delta \mathrm{ppm}$ \\
\hline WT & 1097.19565 & 1097.1932 & 2.2 \\
\hline $\mathrm{C} 22 \mathrm{~S}$ & 1091.86993 & 1091.8680 & 1.8 \\
\hline C26S & 1091.86993 & 1091.8699 & 0.0 \\
\hline${ }^{13} \mathrm{C}-\mathrm{QmpA}$ & 1101.20064 & 1101.20297 & 2.1 \\
\hline \multirow{2}{*}{ Peptide } & \multicolumn{3}{|c|}{-2 Da Product } \\
\hline & {$[\mathrm{M}+3 \mathrm{H}]^{3+}$ calc } & {$[\mathrm{M}+3 \mathrm{H}]^{3+}{ }_{\text {obs }}$} & $\Delta p p m$ \\
\hline WT & 1096.52376 & 1096.5246 & 0.8 \\
\hline $\mathrm{C} 22 \mathrm{~S}$ & 1091.19804 & 1091.1974 & 0.6 \\
\hline C26S & 1091.19804 & 1091.1998 & 1.6 \\
\hline${ }^{13} \mathrm{C}-\mathrm{QmpA}$ & 1100.52876 & 1100.5297 & 0.9 \\
\hline \multirow{2}{*}{ Peptide } & \multicolumn{3}{|c|}{-4 Da Product } \\
\hline & {$[\mathrm{M}+3 \mathrm{H}]^{3+}$ calc } & {$[\mathrm{M}+3 \mathrm{H}]^{3+}{ }_{\text {obs }}$} & $\Delta p p m$ \\
\hline WT & 1095.85188 & 1095.85254 & 0.6 \\
\hline $\mathrm{C} 22 \mathrm{~S}$ & 1090.52616 & - & - \\
\hline C26S & 1090.52616 & - & - \\
\hline${ }^{13} \mathrm{C}-\mathrm{QmpA}$ & 1099.85687 & 1099.8594 & 2.3 \\
\hline
\end{tabular}


Table S3. HR-MS/MS data for the WT QmpA -4 Da product upon reaction with QmpB.

\begin{tabular}{|c|c|c|c|}
\hline Ion & Calc $\mathbf{m} / \mathbf{z}$ & Obs $\mathbf{m} / \mathbf{z}$ & $\Delta \mathbf{p p m}$ \\
\hline $\mathbf{b}_{2}{ }^{+1}$ & 231.11622 & 231.1167 & 2.1 \\
\hline $\mathbf{b}_{3}{ }^{+1}$ & 330.18463 & 330.1855 & 2.6 \\
\hline $\mathbf{b}_{5}{ }^{+1}$ & 545.27524 & 545.2740 & 2.3 \\
\hline $\mathbf{b}_{14}{ }^{+2}$ & 804.40857 & 804.4148 & 7.7 \\
\hline $\mathbf{b}_{15}{ }^{+2}$ & 860.9506 & 860.9492 & 1.6 \\
\hline $\mathbf{b}_{16}{ }^{+2}$ & 924.97989 & 924.9797 & 0.2 \\
\hline $\mathbf{b}_{17}{ }^{+2}$ & 975.50373 & 975.5059 & 2.2 \\
\hline $\mathbf{b}_{18}{ }^{+2}$ & 1011.02228 & 1011.0255 & 3.2 \\
\hline $\mathbf{b}_{19}{ }^{+2}$ & 1061.54612 & 1061.5438 & 2.2 \\
\hline $\mathbf{b}_{20}{ }^{+2}-2 \mathbf{H}$ & 1117.56009 & 1117.5697 & 8.6 \\
\hline $\mathbf{b}_{21}{ }^{+2}-2 \mathbf{H}$ & 1191.09429 & 1191.09802 & 3.1 \\
\hline $\mathbf{b}_{22}{ }^{+2}-2 \mathbf{H}$ & 1242.59888 & 1242.5973 & 1.3 \\
\hline $\mathbf{b}_{23}{ }^{+2}-2 \mathbf{H}$ & 1292.13309 & 1292.1386 & 4.3 \\
\hline $\mathbf{b}_{24}{ }^{+2}-4 \mathbf{H}$ & 1348.14706 & 1348.1495 & 1.8 \\
\hline $\mathbf{b}_{25}{ }^{+2}-4 \mathbf{H}$ & 1404.68909 & 1404.7015 & 8.8 \\
\hline $\mathbf{b}_{26}{ }^{+2}-4 \mathbf{H}$ & 1456.19368 & 1456.2025 & 6.1 \\
\hline $\mathbf{b}_{27}{ }^{+2}-4 \mathbf{H}$ & 1520.22297 & 1520.2224 & 0.4 \\
\hline $\mathbf{b}_{28}{ }^{+2}-4 \mathbf{H}$ & 1585.74321 & 1585.7308 & 7.8 \\
\hline $\mathbf{y}_{2}{ }^{+1}$ & 247.11114 & 247.1120 & 3.5 \\
\hline $\mathbf{y}_{3}{ }^{+1}$ & 375.16971 & 375.1731 & 9.0 \\
\hline $\mathbf{y}_{6}{ }^{+1}-2 \mathbf{H}$ & 703.29089 & 703.2860 & 7.0 \\
\hline $\mathbf{y}_{11}{ }^{+1}-2 \mathbf{H}$ & 1265.51251 & 1265.5179 & 4.3 \\
\hline $\mathbf{y}_{13}{ }^{+1}-2 \mathbf{H}$ & 1437.5973 & 1437.5895 & 5.4 \\
\hline $\mathbf{y}_{28}{ }^{+3}-4 \mathbf{H}$ & 1052.1722 & 1052.1621 & 9.6 \\
\hline
\end{tabular}


Table S4. HR-MS/MS data for the C22S-QmpA product upon reaction with QmpB.

\begin{tabular}{|c|c|c|c|}
\hline Ion & Calc $\mathbf{m} / \mathbf{z}$ & Obs $\mathbf{m} / \mathbf{z}$ & $\Delta$ ppm \\
\hline $\mathbf{b}_{2}{ }^{+1}$ & 231.11622 & 231.115 & 5.3 \\
\hline $\mathbf{b}_{3}{ }^{+1}$ & 330.18463 & 330.1843 & 1.0 \\
\hline $\mathbf{b}_{14}{ }^{+2}$ & 804.40857 & 804.4073 & 1.6 \\
\hline $\mathbf{b}_{15}{ }^{+2}$ & 860.9506 & 860.9498 & 0.9 \\
\hline $\mathbf{b}_{16}{ }^{+2}$ & 924.97989 & 924.9775 & 2.6 \\
\hline $\mathbf{b}_{17}{ }^{+2}$ & 975.50373 & 975.5019 & 1.9 \\
\hline $\mathbf{b}_{18}{ }^{+2}$ & 1011.02228 & 1011.0196 & 2.7 \\
\hline $\mathbf{b}_{19}{ }^{+2}$ & 1061.54612 & 1061.5445 & 1.5 \\
\hline $\mathbf{b}_{20}{ }^{+2}$ & 1118.56759 & 1118.5667 & 0.8 \\
\hline $\mathbf{b}_{21}{ }^{+2}$ & 1192.10179 & 1192.0987 & 2.6 \\
\hline $\mathbf{b}_{22}{ }^{+2}$ & 1235.61781 & 1235.6145 & 2.7 \\
\hline $\mathbf{b}_{23}{ }^{+2}$ & 1285.15201 & 1285.1511 & 0.7 \\
\hline $\mathbf{b}_{24}{ }^{+2}-2 \mathrm{H}$ & 1341.16598 & 1341.1672 & 0.9 \\
\hline $\mathbf{b}_{25}{ }^{+2}-2 \mathrm{H}$ & 1397.70801 & 1397.7082 & 0.1 \\
\hline $\mathbf{b}_{27}{ }^{+2}-2 \mathrm{H}$ & 1513.24189 & 1513.2417 & 0.1 \\
\hline $\mathbf{b}_{28}{ }^{+2}-2 \mathrm{H}$ & 1578.76213 & 1578.7607 & 0.9 \\
\hline $\mathbf{y}_{1}{ }^{+1}$ & 116.07065 & 116.06 & 91.8 \\
\hline $\mathbf{y}_{2}{ }^{+1}$ & 247.11114 & 247.11 & 4.6 \\
\hline $\mathbf{y}_{3}{ }^{+1}$ & 375.16971 & 375.1717 & 5.3 \\
\hline $\mathbf{y}_{6}{ }^{+1}-2 \mathbf{H}$ & 703.29089 & 703.29 & 1.3 \\
\hline
\end{tabular}


Table S5. HR-MS/MS data for the C26S-QmpA product upon reaction with QmpB.

\begin{tabular}{|c|c|c|c|}
\hline Ion & Calc $\mathrm{m} / \mathrm{z}$ & Obs $\mathrm{m} / \mathrm{z}$ & $\Delta p p m$ \\
\hline$b_{2}^{+1}$ & 231.11622 & 231.1088 & 32.1 \\
\hline$b_{3}^{+1}$ & 330.18463 & 330.1852 & 1.7 \\
\hline$b_{7}^{+1}$ & 787.41313 & 787.4150 & 2.4 \\
\hline$b_{11}+1$ & 1308.66169 & 1308.6574 & 3.3 \\
\hline$b_{12}+2$ & 719.3558 & 719.3581 & 3.2 \\
\hline$b_{14}+2$ & 804.40857 & 804.4073 & 1.6 \\
\hline$b_{15}{ }^{+2}$ & 860.9506 & 860.9492 & 1.6 \\
\hline$b_{16}{ }^{+2}$ & 924.97989 & 924.9778 & 2.3 \\
\hline$b_{17}+2$ & 975.50373 & 975.502 & 1.8 \\
\hline$b_{18}+2$ & 1011.02228 & 1011.0199 & 2.4 \\
\hline$b_{19}{ }^{+2}$ & 1061.54612 & 1061.5442 & 1.8 \\
\hline$b_{20}+2-2 H$ & 1117.56009 & $1117.5630^{* *}$ & 2.6 \\
\hline $\mathrm{b}_{21}{ }^{+2}-2 \mathrm{H}$ & 1191.09429 & 1191.0854 & 7.5 \\
\hline $\mathrm{b}_{22}{ }^{+2}-2 \mathrm{H}$ & 1242.59888 & 1242.5864 & 10 \\
\hline$b_{23}{ }^{+2}-2 \mathrm{H}$ & 1292.13309 & 1292.1354 & 1.8 \\
\hline$b_{24}{ }^{+2}-2 H$ & 1349.15456 & 1349.1434 & 8.3 \\
\hline$b_{25}{ }^{+2}-2 \mathrm{H}$ & 1405.69659 & 1405.6955 & 0.8 \\
\hline$b_{26}{ }^{+2}-2 \mathrm{H}$ & 1449.2126 & 1449.2065 & 4.2 \\
\hline$b_{27}+2-2 H$ & 1513.24189 & 1513.2324 & 6.3 \\
\hline$y_{1}+1$ & 116.07065 & 116.0491 & 185.7 \\
\hline $\mathrm{y}_{2}^{+1}$ & 247.11114 & 247.1055 & 22.8 \\
\hline$y_{3}^{+1}$ & 375.16971 & 375.1719 & 5.8 \\
\hline $\mathrm{y}_{4}^{+1}$ & 462.20174 & 462.2059 & 9.0 \\
\hline
\end{tabular}


Figure S1. UV-vis absorption spectrum of purified and reconstituted QmpB. The spectrum displays the hallmark $\sim 320 \mathrm{~nm}$ shoulder and a broad $400 \mathrm{~nm}$ absorption features, which are symptomatic of [4Fe-4S] clusters.

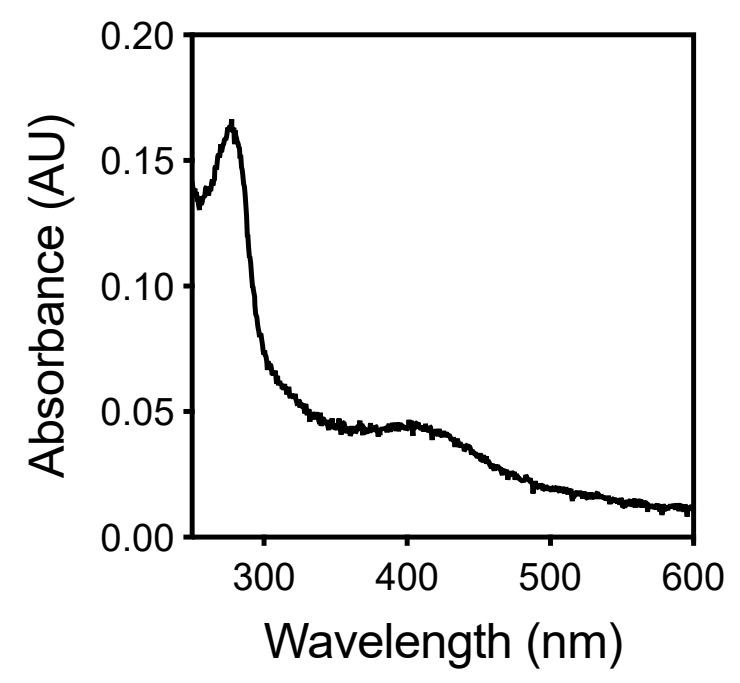

Figure S2. SPASM domain alignment highlighting auxiliary [4Fe4S] cluster-binding cysteine residues in CteB and SuiB (pdb id: 5WHY and 5V1Q). Red cysteines bind Auxl and blue cysteines bind Auxll in CteB and SuiB. Similarly positioned cysteines are highlighted in QmpB, which may bind auxiliary clusters.

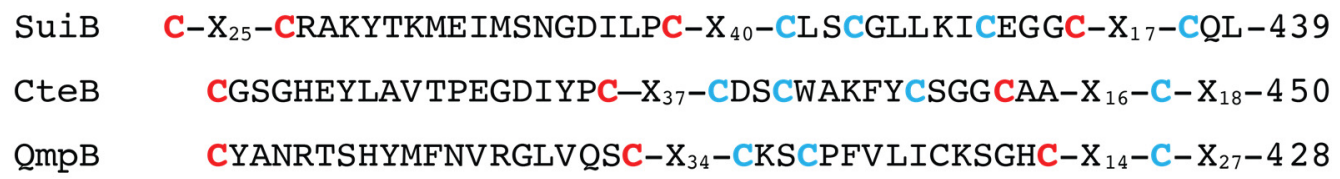


Figure S3. Time-dependent formation of 5'-deoxyadenosine in the reaction of $S$. suis QmpB with SAM and reductant $\left(\mathrm{Na}_{2} \mathrm{~S}_{2} \mathrm{O}_{4}\right)$ in the absence of $\mathrm{QmpA}$ substrate. $0.6 \mathrm{nmol}$ of $\mathrm{QmpB}$ was used at each timepoint. 5'-deoxyadenosine was quantified using a standard curve. The average of two independent measurements are shown at each time point; bars represent standard error.

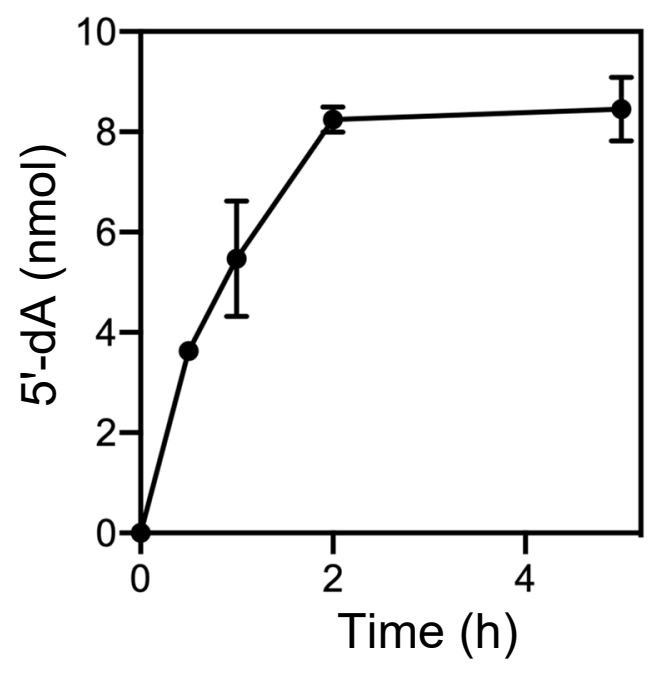

Figure S4. HR-MS/MS data for C28S-NxxcA following reaction with NxxcB. No fragments were observed within the $\beta$-thioether macrocycle.

NxxcB product MS/MS

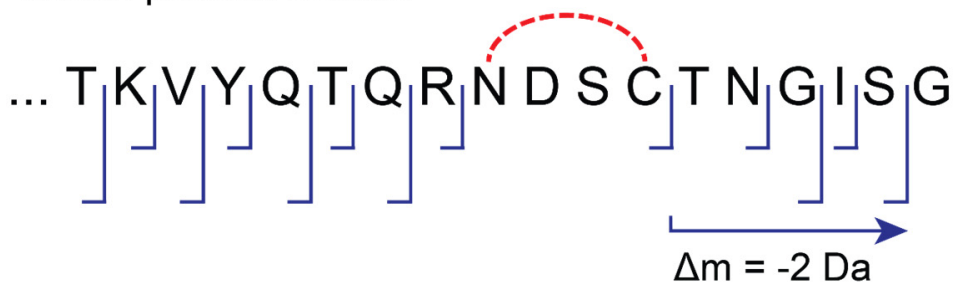


Figure S5. HR-MS analysis of modified ${ }^{13} \mathrm{C}-\mathrm{QmpA}$ after reaction with $\mathrm{QmpB}$. (A) Extracted ion chromatogram of the $\mathrm{QmpB}$ products (-2 $\mathrm{Da}$ in red; $-4 \mathrm{Da}$ in blue) and remaining unmodified substrate (black). (B) Mass spectra of modified and unmodified ${ }^{13} \mathrm{C}$ QmpA products. The triply-ionized mass envelope is shown with the $-2 \mathrm{Da}$ masses highlighted in red and -4 Da masses highlighted in blue.
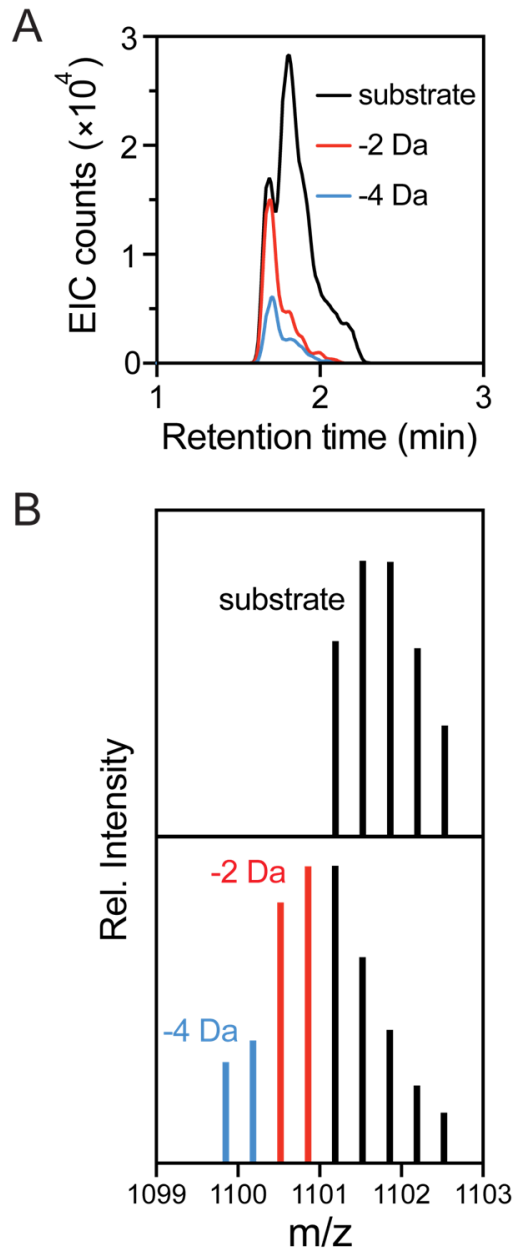
Figure S6. NMR spectra of unmodified ${ }^{13} \mathrm{C}-\mathrm{QmpA}$ in $200 \mathrm{mM} \mathrm{NH} \mathrm{HCO}_{3}$ and $\mathrm{D}_{2} \mathrm{O}$. Shown are $(A){ }^{1} \mathrm{H},(B){ }^{13} \mathrm{C}$, and $(\mathrm{C}) \mathrm{HSQC}$ spectra. The absence of the thioether product crosspeak at $C \beta / H \beta$ is indicated with a rectangle in the HSQC spectrum (C).

A

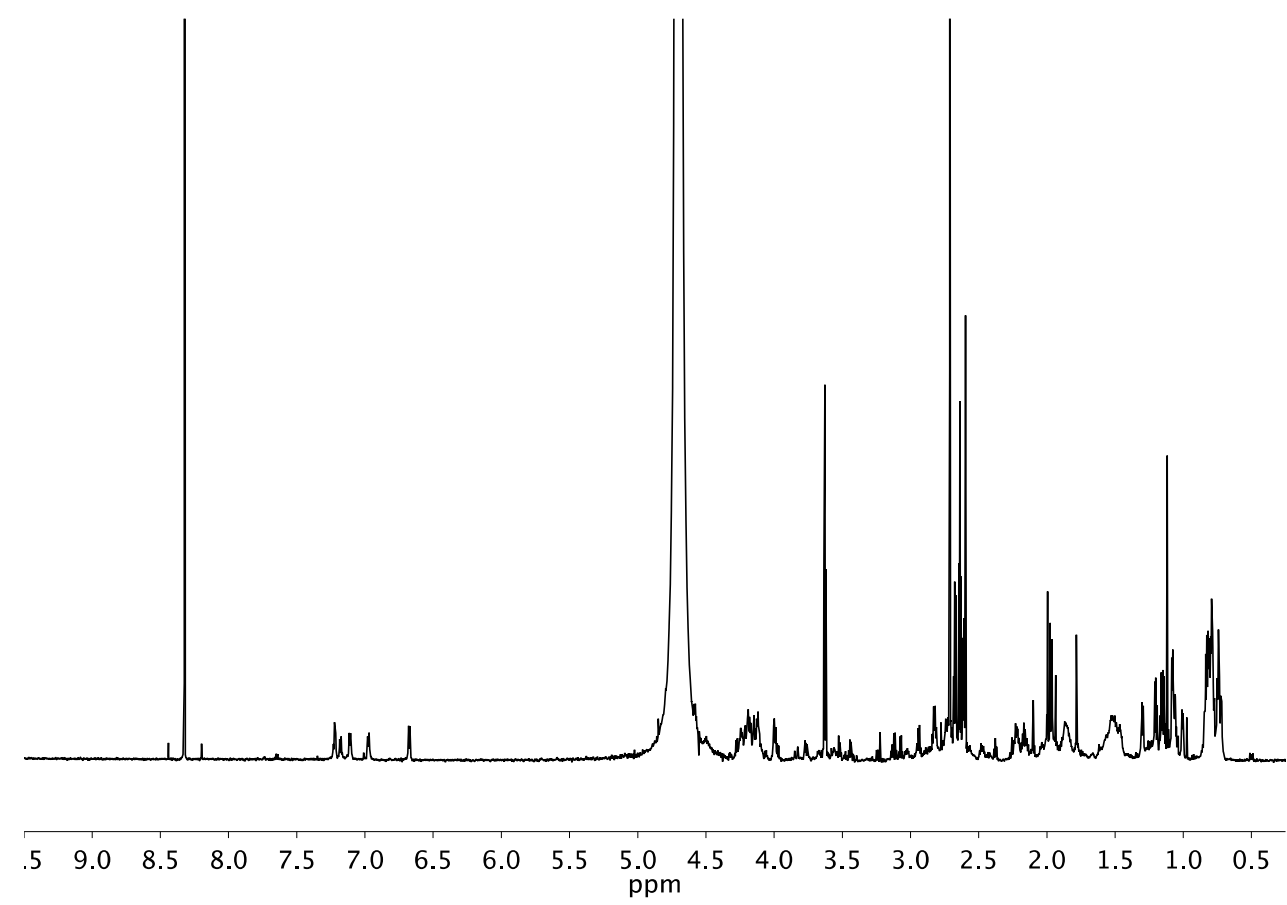

B

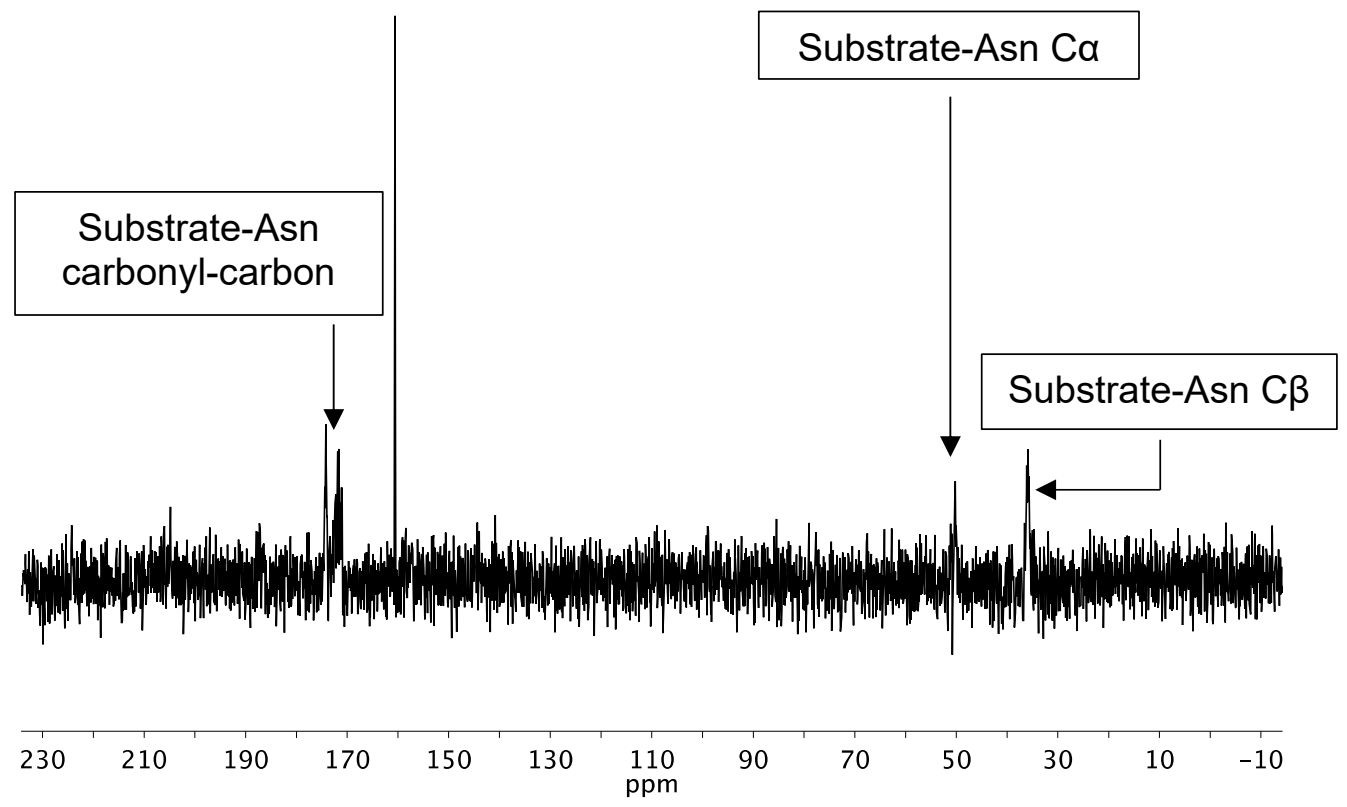




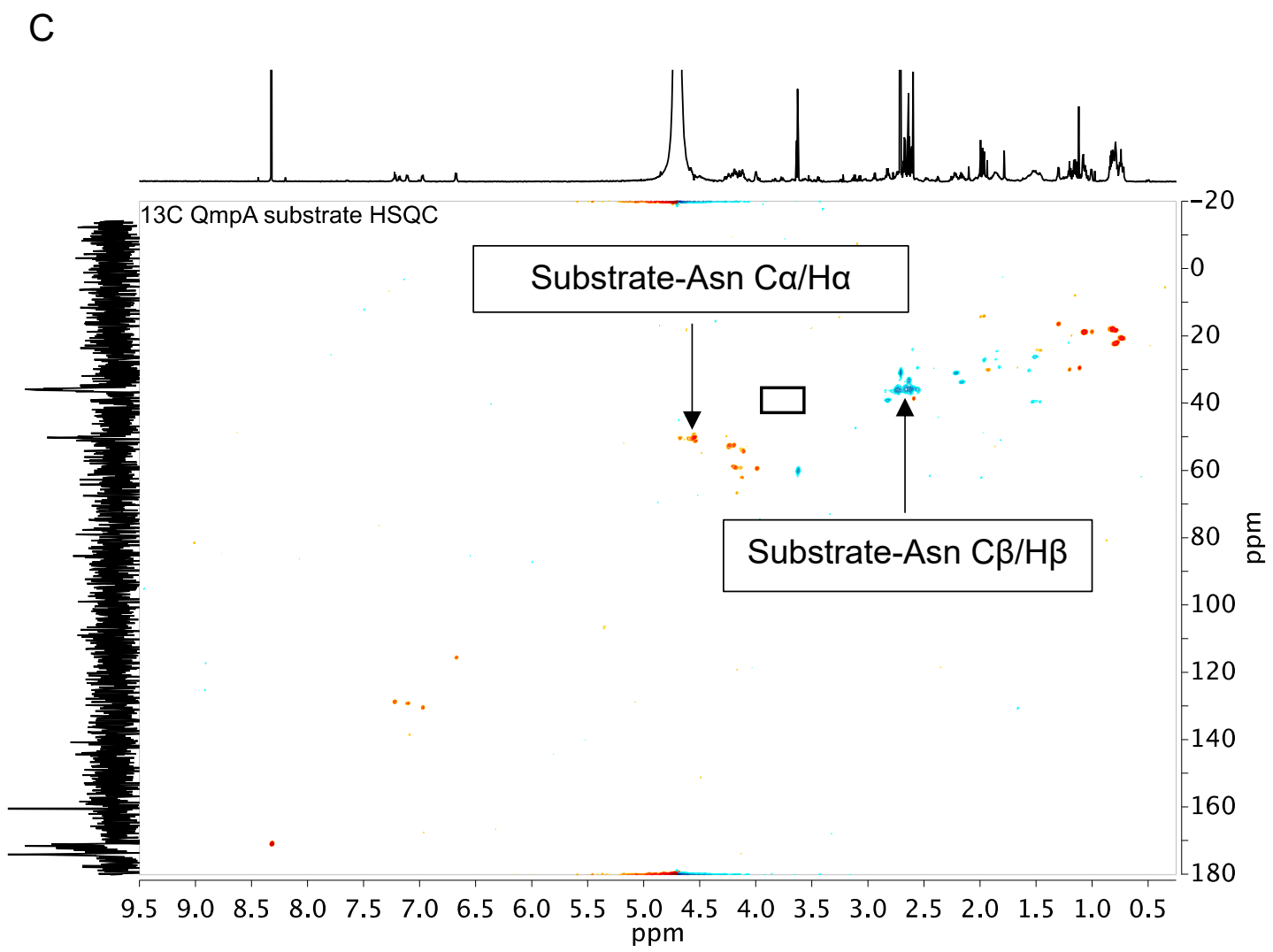


Figure S7. NMR spectra of ${ }^{13} \mathrm{C}-\mathrm{QmpA}$ substrate/product mixture in $200 \mathrm{mM} \mathrm{NH}_{4} \mathrm{HCO}_{3}$ and $\mathrm{D}_{2} \mathrm{O}$ following reaction with $\mathrm{QmpB}$. Shown are $(\mathrm{A}){ }^{1} \mathrm{H},(\mathrm{B}){ }^{13} \mathrm{C}$, and $(\mathrm{C}) \mathrm{HSQC}$ spectra. Note, due to poor conversion to product, the ${ }^{13} \mathrm{C}$-Asn product peaks are not observable in the $1 \mathrm{D}-{ }^{13} \mathrm{C}$ spectrum. They can, however, be identified in the HSQC spectrum.

A

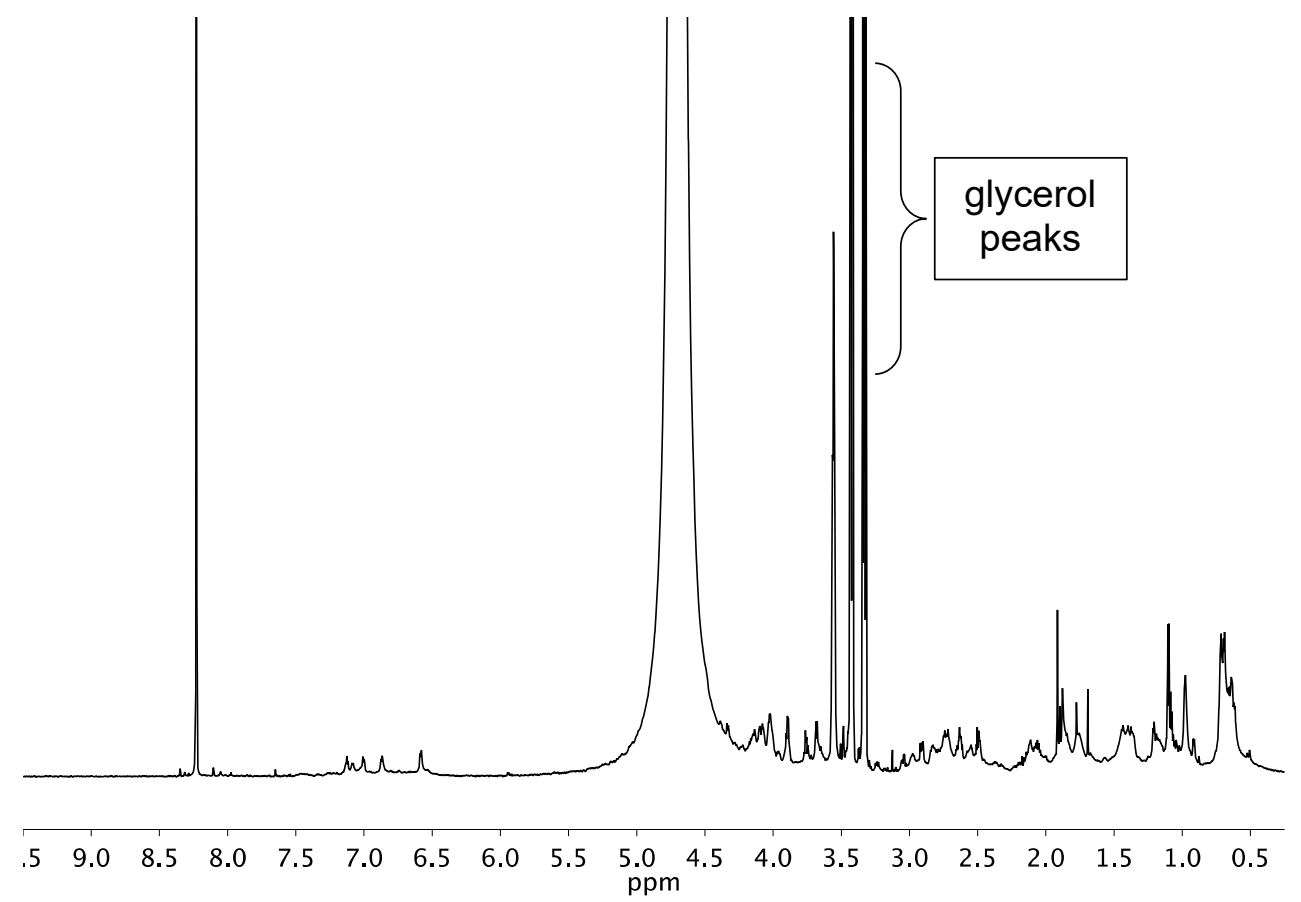

B

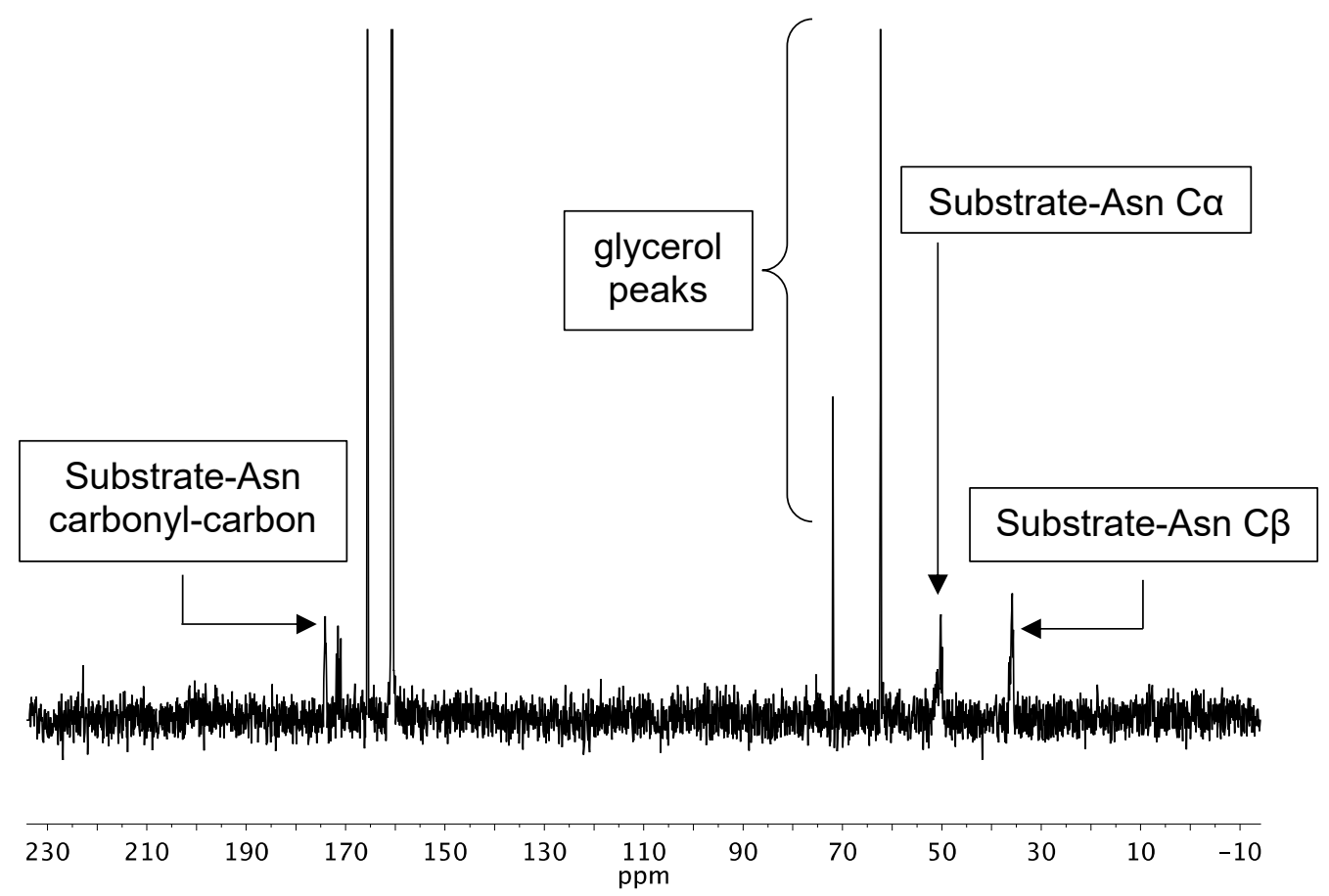




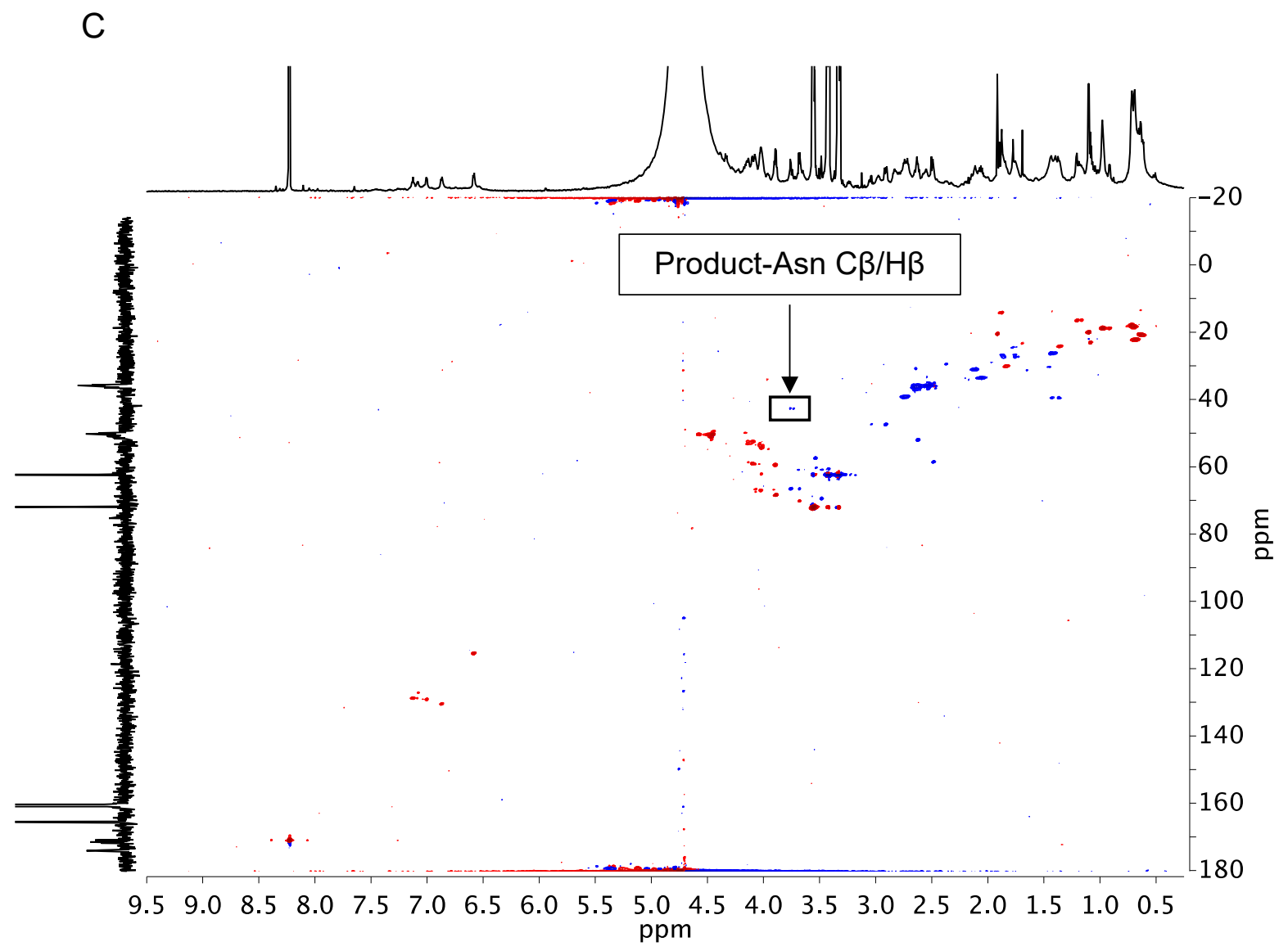

